\title{
Enrichment of colorectal cancer stem cells through epithelial-mesenchymal transition via $\mathrm{CDH} 1$ knockdown
}

\author{
JUN YE*, DANG WU*, JINWEN SHEN, PIN WU, CHAO NI, JING CHEN, \\ JING ZHAO, TAO ZHANG, XIAOLEI WANG and JIAN HUANG
}

\begin{abstract}
Cancer Institute (Key Laboratory for Cancer Prevention and Intervention, China National Ministry of Education, Key Laboratory of Molecular Biology in Medical Sciences, Zhejiang), Second Affiliated Hospital, Zhejiang University School of Medicine, Hangzhou, Zhejiang 310009, P.R. China
\end{abstract}

Received February 2, 2012; Accepted May 23, 2012

DOI: $10.3892 / \mathrm{mmr} .2012 .938$

\begin{abstract}
Cancer stem cells (CSCs) are considered to be the origin of cancer relapse and metastasis. The better understanding of CSCs, including CSCs in human colorectal cancer (CRC), may facilitate prevention and treatment. This study aimed to establish a CSC enrichment model via the induction of epithelial-mesenchymal transition (EMT) in CRC cells. We established an EMT model using the SW480 CRC cell line by CDH1 knockdown using shRNA interference. CD $24^{+} \mathrm{CD} 44^{+}$ cells were enriched in the $C D H 1$ knockdown cells. The cells exhibited mesenchymal morphology and expressed high levels of EMT-related proteins, which confirmed that these cells had undergone EMT. Our results further showed that the proliferation rate of the transfected cells was reduced, whereas their colony-forming capacity and tumorigenesis in vivo was significantly enhanced compared to the control cells. In conclusion, these cells were highly enriched CSCs (compared to normal CSCs) and may be used as a stable model for cancer research and anticancer drug screening.
\end{abstract}

\section{Introduction}

Colorectal cancer (CRC) is the third most common type of cancer in men and the second in women worldwide $(1,2)$. It is one of the leading causes of cancer-related mortality due to therapy resistance (3). A better understanding of the molecular mechanisms underlying CRC progression is essential for the prevention and treatment of advanced CRC.

A novel paradigm in tumor biology suggests that cancer growth is driven by stem-like cells within a tumor. Growing

Correspondence to: Professor Jian Huang, Cancer Institute, Second Affiliated Hospital, Zhejiang University School of Medicine, 88 Jiefang Road, Hangzhou, Zhejiang 310009, P.R. China

E-mail: drhuangjian@zju.edu.cn

${ }^{*}$ Contributed equally

Key words: colorectal cancer, cancer stem cells, epithelialmesenchymal transition, E-cadherin evidence suggests that human cancers are stem cell diseases. Recent data support the hypothesis that cancer stem cells (CSCs) exist in human cancers, including CRC (4). CSCs are a subpopulation of cells within a tumor that can self-renew, drive tumor growth and recurrence and are resistant to many current anticancer treatments $(5,6)$.

Epithelial-mesenchymal transition (EMT) plays a crucial role in the differentiation of multiple tissues and organs, and is a key developmental program that is often activated during cancer invasion and metastasis (7-9). Previous studies have provided morphological evidence that EMT occurs at the invasive fronts of human tumors, including CRC, and EMT occurring at the tumor-host interface is thought to enhance metastasis $(10,11)$.

During the process of EMT, epithelial cells undergo a phenotypic switch, giving rise to a fibroblastoid phenotype. However, since this 'transformation' is reversible and mesenchymal cells revert to epithelial cells via mesenchymalepithelial transition (MET) (12), the mesenchymal state is associated with the capacity of cells to prevent apoptosis and senescence, and contributes to immunosuppression, migration to distant organs and maintaining stemness $(7,8)$. The induction of EMT in normal and cancer cell populations renders them more resistant to chemotherapeutic drugs (5).

E-cadherin (encoded by $\mathrm{CDH1}$ ) is a transmembrane glycoprotein localized in the apical adherens junction typically found in epithelial cells, and plays an important role in maintaining the structural integrity of epithelial sheets (12). E-cadherin is an important molecule in cancer progression and EMT process. Indeed, E-cadherin perturbation in mammalian cell systems is sufficient to trigger EMT (13). The inhibition of E-cadherin expression has been reported in several types of cancer, including advanced colorectal cancer, oesophageal adenocarcinoma, gastric cancer, vulvar squamous cell carcinoma and pancreatic cancer $(9,12,14)$. The loss of E-cadherin is considered to augment cellular dissemination and tumor metastasis (12).

In this study, we demonstrate that the knockdown of E-cadherin in the SW480 colorectal cancer cell line leads to significant EMT-like alterations and acquirement of most the properties of CSCs. The aim of this study was to create a model of CSC enrichment for cancer study, especially for screening anti-CSC chemotherapeutic drugs. 


\section{Materials and methods}

Cell culture. The SW480 human colorectal cancer cell line was purchased from the cell bank of the China Academy of Medical Science (China) and cultured in Leibovitz L15 medium (Gibco) supplemented with $10 \%$ fetal bovine serum (FBS; Gibco). The cells were then maintained at $37^{\circ} \mathrm{C}$ with $5 \% \mathrm{CO}_{2}$.

Gene knockdown. Gene knockdown was achieved by transfecting SW480 cells with $C D H 1$ shRNA-pSilencer 4.1-CMV using Lipofectamine 2000 (Invitrogen) in accordance with the manufacturer's instructions. After transfection, the cells were maintained under G418 selection pressure at a concentration of $600 \mu \mathrm{g} / \mathrm{ml}$ for 14 days. Limited dilution assay was performed, and 10 clones with $>90 \%$ knockdown efficiency verified by qRT-PCR were selected (Fig. 1A), expanded and then cultured together. The cells transfected with $C D H 1$ shRNA-pSilencer 4.1-CMV were annotated as SW480-shE. The cells transfected with pSilencer 4.1-CMV were annotated as SW480-shC.

qRT-PCR analysis. Total RNA was extracted from the cultured cells using the RNeasy mini kit (Qiagen) and treated with RNase-free DNase I (Qiagen) according to the manufacturer's instructions. The RNA from SW480-shC and SW480-shE cells was reverse-transcribed into cDNA with the Reverse Transcriptase M-MLV (Promega). The expression of $C D H 1$ was measured using the SYBR-Green PCR Master Mix (Applied Biosystems), on the StepOnePlus system (Applied Biosystems). The $C D H 1$ levels were normalized to GAPDH expression.

The primers for qRT-PCR analysis were the following: $C D H 1$ forward, 5'-GCTCACATTTCCCAACTC-3' and reverse, 5'-GTCACCTTCAGCCATCC-3'; GAPDH forward, 5'-CTT AGCACCCCTGGCCAAG-3' and reverse, 5'-GATGTTCTGG AGAGCCCCG-3'.

Western blot analysis. SW480-shC and SW480-shE cells were lysed using M-PER Mammalian Protein Extraction Reagent (Thermo) supplemented with protease inhibitor cocktail(Sigma). After blocking with 5\% non-fat milk in TBST for $60 \mathrm{~min}$, the membranes were incubated with primary antibodies dissolved in $5 \%$ bovine serum albumin (BSA) in TBST overnight at $4^{\circ} \mathrm{C}$. The following primary antibodies were used: anti-human-Ecadherin (Cell Signaling Technology), anti-human-N-cadherin (Epitomics) and anti-human-Vimentin (Epitomics) at a 1:2,000, 1:5,000 or 1:1,000 dilution, respectively. The membranes were washed with TBST for $5 \mathrm{~min} 3$ times and then incubated for $1 \mathrm{~h}$ at room temperature with secondary antibody $(1: 3,000$, swine anti-rabbit IgG/HRP; Gene Tech) dissolved in 5\% non-fat milk in TBST. Human GAPDH (KangChen) was used as an internal reference at a 1:5,000 dilution.

Proliferation assays. SW480-shC and SW480-shE cells were seeded at a density of $3 \times 10^{3}$ cells/well in a 96-well plate containing $0.2 \mathrm{ml}$ Leibovitz L15 medium (Gibco) with $10 \%$ FBS. Subsequently, $20 \mu 1$ 3-(4,5-dimethylthiazol-2-yl)-5(3-carboxymethoxyphenyl)-2-(4-sulfophenyl)-2H-tetrazolium, inner salt (MTS; Promega) reagent was added to each well and the cells were incubated at $37^{\circ} \mathrm{C}$ for $4 \mathrm{~h}$. The OD values were measured at $490 \mathrm{~nm}$ on a microplate reader (Bio-Rad, Hercules, CA, USA) and assessed every day for up to 7 days.
Two-dimensional (2D) and three-dimensional (3D) colony formation assay. The growth ability of SW480-shC and SW480-shE cells was examined using 2D colony formation assay. Approximately 500 SW480-shC or SW480-shE cells were seeded into each well of a 6 -well plate. After incubation at $37^{\circ} \mathrm{C}$ for 14 days, the cells were washed with PBS twice, fixed with methanol and then stained with $0.1 \%$ crystal violet. The number of colonies containing $>30$ cells was counted under a microscope. In 3D colony formation assay, 300 cells of each cell line were seeded into each well of an ultra-low attachment 6-well plate (Corning) and cultured in serum-free DMEM/F12 (Gibco) containing $1 \mu \mathrm{g} / \mathrm{ml}$ insulin (Haotian Biotechnology), $20 \mathrm{ng} / \mathrm{ml} \mathrm{bFGF} \mathrm{(Invitrogen),} 20 \mathrm{ng} / \mathrm{ml} \mathrm{EGF}$ (Invitrogen), 0.1\% BSA (Haotian Biotechnology) and 2\% B27 (Invitrogen). After 14 days of incubation, the number of colospheres containing $>30$ cells was counted. Experiments were performed in triplicate.

Flow cytometry analysis. SW480-shC and SW480-shE cells were trypsinized and fixed in $70 \%$ ethanol. DNA content of incorporated propidium iodide (PI) was scanned on a flow cytometer. The cell populations in G0/G1, S and G2/M phases were determined using the ModFit LT software.

CD24 and CD44 are widely used for isolating CSCs from solid tumors, including CRC $(14,15)$. Combining these 2 markers enhances the purity of CSCs. For comparing the proportion of $\mathrm{CD} 24^{+} / \mathrm{CD} 44^{+}$cells in the SW480-shC and SW480-shE cell populations, cells were trypsinized, washed with phosphate-buffered saline (PBS) and resuspended in 2\% FBS in PBS at $1 \times 10^{6}$ cells $/ 100 \mu$ l. Each suspension was incubated with mouse anti-human CD24-FITC (BD Pharmingen) and CD44-APC antibodies (Miltenyi Biotec) for $20 \mathrm{~min}$ at $4^{\circ} \mathrm{C}$. The isotype control and blank control were set in the analysis. Then, the cells were washed twice with PBS and filtered on a 300 mesh screen to exclude the cell mass. Cells labeled with $\mathrm{CD} 24^{+} / \mathrm{CD} 44^{+}$markers were finally analyzed using a FACSCanto II flow cytometer (BD Biosciences). The FACSDiva software was used to quantify the fluorescence signals and to set the logical electronic-gating parameters.

Tumorigenesis assay. SW480-shC and SW480-shE cells $\left(1 \times 10^{5}\right)$ suspended in $200 \mu 1$ Leibovitz L15 medium were implanted into the backs of 4-week-old female nude mice. The mice were either injected with SW480-shC or SW480-shE cells to analyze their ability to initiate tumor xenografts. Nude mice were bred and maintained under defined conditions at the Laboratory Animal Research Center of Zhejiang Chinese Medicine University under conditions approved by the local animal care committee.

Statistical analysis. Unless otherwise indicated, all the results were analyzed by a two-tailed unpaired Student's t-test.

\section{Results}

Knockdown of CDH1 induces EMT in SW480 cells. We established a cell line model by $C D H 1$ knockdown in SW480 cells. SW480 cells were transfected with $C D H 1$ shRNA-pSilencer 4.1-CMV (shE vector) or pSilencer 4.1-CMV (shC vector). Western blot analysis showed that E-cadherin protein expression 
$\mathbf{A}$

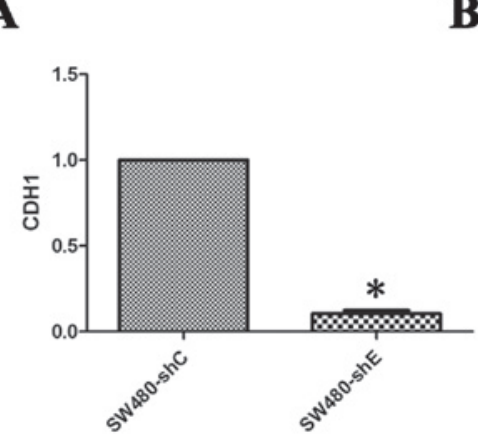

$\mathbf{C}$

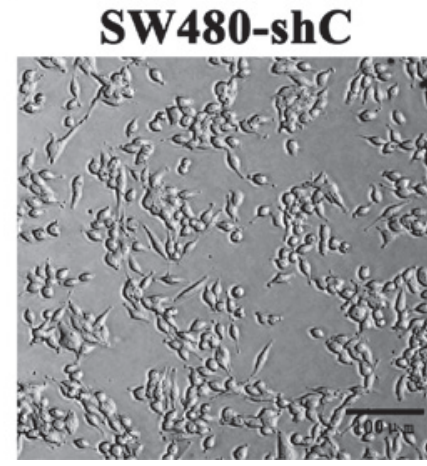

B

E-cadherin

N-cadherin

Vimentin

GAPDH

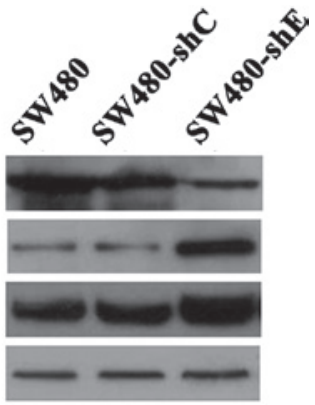

Figure 1. $C D H 1$ knowdown induced EMT. (A) mRNA expression levels of $C D H 1$ in SW480 cells treated with $C D H 1$ knockdown. (B) Protein levels of E-cadherin, N-cadherin and Vimentin in SW480 cells after $C D H 1$ knockdown. (C) Morphological changes in SW480-shE cells. Images were taken under a microscope (magnification, x200).

$\mathbf{A}$

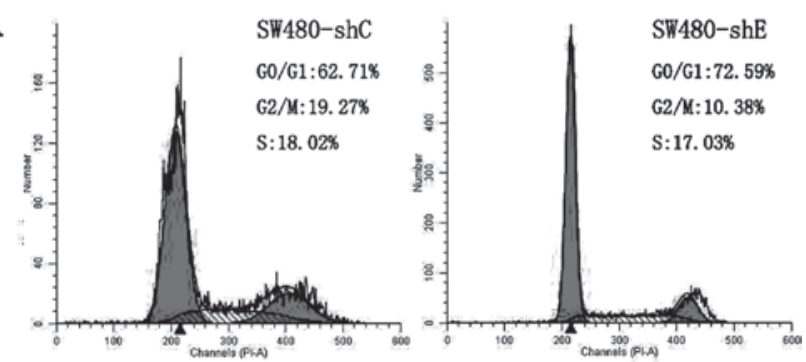

B

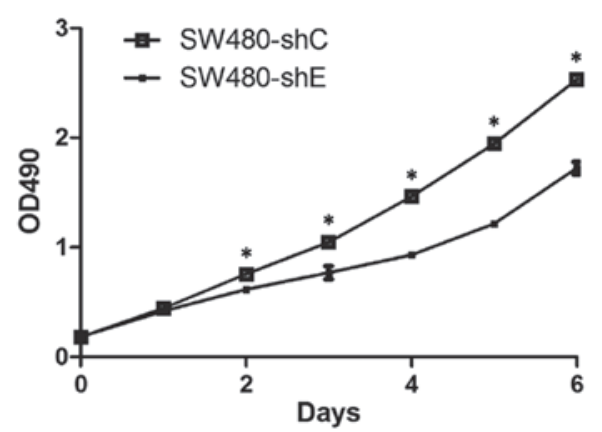

Figure 2. Knockdown of $C D H 1$ in SW480 cells attenuated cell proliferation in vitro. (A) Cell cycle analysis of SW480-shC and SW480-shE cells. (B) Knockdown of CDH1 in SW480 cells attenuated cell proliferation. Data are presented as the means \pm SEM $\left({ }^{*} \mathrm{P}<0.05\right)$.

was downregulated when the shE vector was transfected into the SW480 cells successfully (Fig. 1B). By contrast, the transfection of the shC vector had no effect on protein expression.
SW480-shE cells presented with mesenchymal morphology with disrupted intercellular adhesion and a reorganized cytoskeleton into an EMT-like formation (Fig. 1C). The results obtained from western blot analysis showed that the SW480-shE cells expressed high levels of mesenchymal cell marker proteins, including N-cadherin and Vimentin (Fig. 1B). These results collectively proved that SW480-shE cells had undergone EMT.

SW480-shE cells have reduced cellular proliferation. The cell cycles of the SW480-shC and SW480-shE cells were then analyzed by flow cytometry and PI staining. The SW480-shE cells had a significantly increased number of cells in the $\mathrm{G} 0$ / G1 phase compared to the SW480-shC cells (72.59 vs. 62.71\%) (Fig. 2A).

We compared the proliferation rate between SW480-shC and SW480-shE cells using MTS analysis. As shown in the growth curve, the proliferative ability of the SW480-shE cells was significantly inhibited compared to the SW480-shC cells over a 7-day test period (Fig. 2B). The results demonstrated that the knockdown of $\mathrm{CDH} 1$ reduced the cell proliferative ability in vitro.

SW480-shE cells have significantly enhanced colony formation. Certain evidence indicates that EMT generates cells with properties of stem cells $(5,7)$. One feature of 'stemness' is the ability to form colonies. In the present study, we examined this hypthesis using colony formation assay. The colonyforming ability of the SW480-shC and SW480-shE cells was examined. The results showed that colonies were visible after 
$\mathbf{A}$

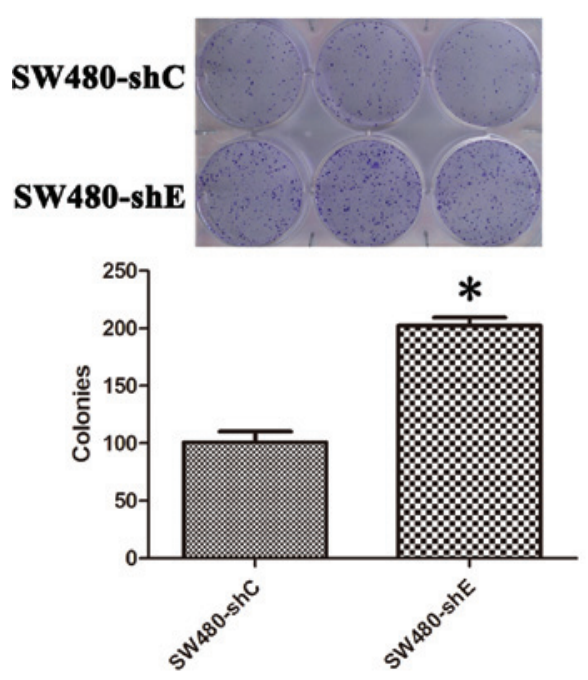

B

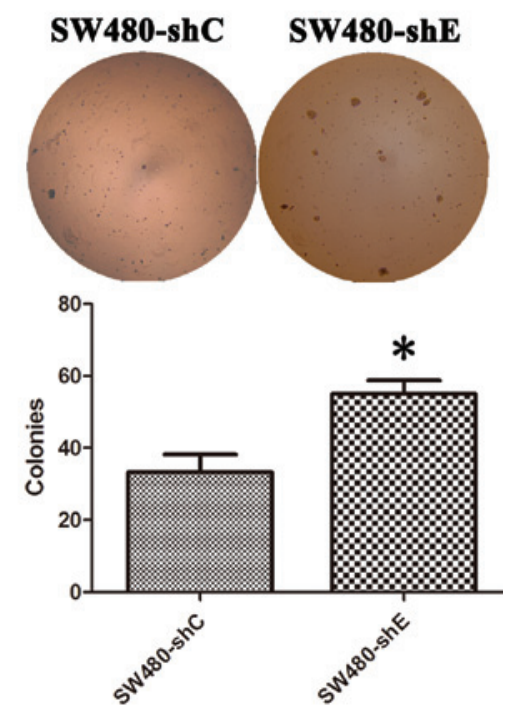

$\mathbf{C}$

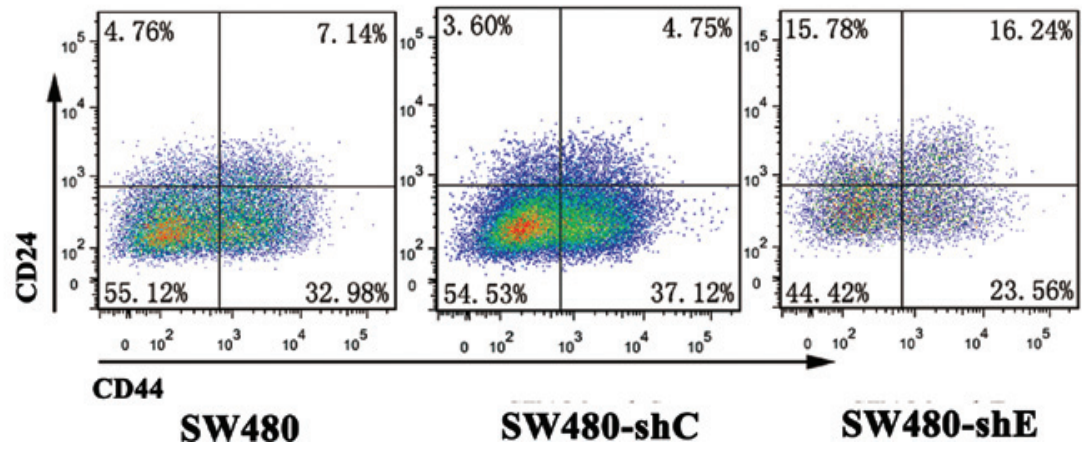

Figure 3. Knockdown of $\mathrm{CDH} 1$ in SW480 cells enhanced the colony-formating ability and upregulated CD24/CD44 expression. (A) 2D plate colony formation assay. (B) 3D colony formation assay. Data are presented as the means \pm SEM ( $\left.{ }^{*} \mathrm{P}<0.05\right)$. (C) $\mathrm{CD} 24^{+} \mathrm{CD} 44^{+}$cells were enriched in $\mathrm{SW} 480$-shE cells.

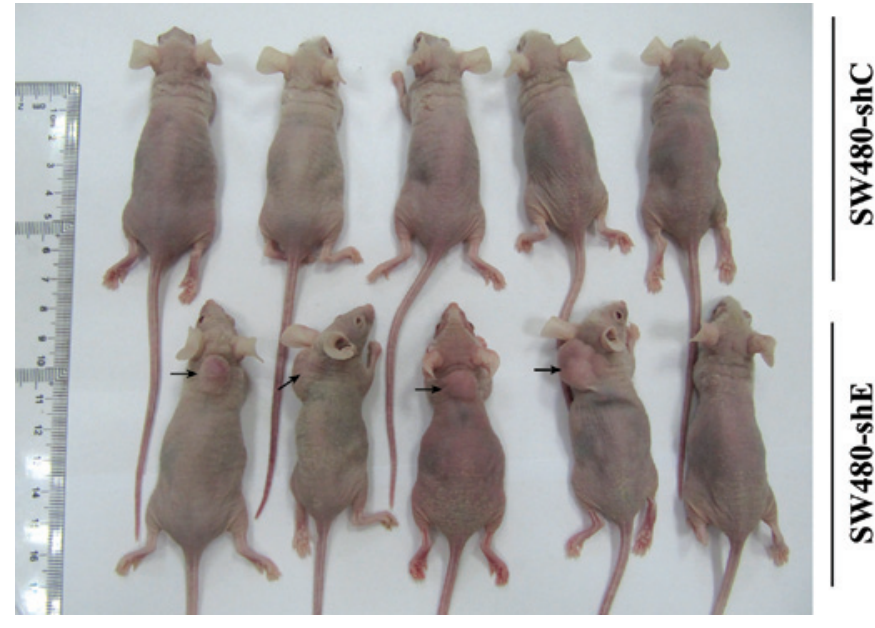

Figure 4. Increased tumorigenic ability of SW480-shE cells. Arrows indicate xenografts.

2 weeks and the number and size of colonies were significantly greater in the SW480-shE cells compared to the SW480-shC cells (Fig. 3A and B).
CD24/CD44 expression is upregulated in SW480-shE cells. CD24 and CD44 are widely accepted surface markers used for isolating CSCs from solid tumors, including CRC (16). We detected the proportion of $\mathrm{CD} 24^{+} / \mathrm{CD} 44^{+}$cells in both the SW480-shC and SW480-shE cells by flow cytometry. The result revealed that the proportion of $\mathrm{CD} 24^{+} / \mathrm{CD} 44^{+}$-expressing cells increased from 4.75 to $16.24 \%$ in the SW480-shE cells, while no significant difference was observed in the SW480-shC cells (Fig. 3C). This result suggested that the knockdown of CDH1 made the SW480 cells more stem-like, giving them the property of 'stemness'.

CDH1 knockdown promotes tumorigenesis. SW480-shC and SW480-shE cells $\left(1 \times 10^{5}\right)$ were injected subcutaneously into the backs of nude mice. The mice were divided into 2 groups: those injected with SW480-shC cells and those injected with SW480-shE cells. In the SW480-shE group, xenograft tumors could be detected in 4 out of 5 mice at 3 weeks after inoculation, while no visible tumor could be observed at 5 weeks in the SW480-shC group. A significant difference in tumor growth was observed between the SW480-shC and SW480-shE xenograft mice (Fig. 4). From these results, it is evident that 
the knockdown of $\mathrm{CDHI}$ gene promoted the tumorigenesis of CRC.

\section{Discussion}

To date, the existence of CSCs has been confirmed in tumors from many organs, including breast, brain, prostate, pancreas, head and neck, colon, lung, skin, liver and ovary $(17,18)$. CSCs are a small subpopulation of cancer cells, which expand the CSC pool and differentiate into cancer progenitor cells by symmetric or asymmetric division (19). CSCs are quiescent, resistant to therapy and responsible for sustaining tumor growth and recurrence $(4,5,20,21)$. Although there have been many advances in CSC research, the origin of CSCs and the differences between CSCs and differentiated cancer cells remain unclear.

There is growing evidence suggesting that EMT plays an important role in tumor development, not only in tumor recurrence, but also in tumor metastasis (22). In CRC, EMT occurs at the invasive front and produces single migratory cells with decreased E-cadherin expression $(8,10)$. Recent evidence shows that cells which undergo EMT acquire stem cell-like properties $(5,7)$. It has been reported that the proportion of CSCs increases in breast cancer cells with the induction of EMT by the knockdown of the human $C D H 1$ gene (5). However, a stable model of CSCs via $C D H 1$ knockdown in CRC has not yet been established.

In this study, we provide evidence to support the hypthesis that EMT participates in CRC progression. As shown in a previous study, non-motile, polarized epithelial cells dissolved their cell-cell junctions and converted into individual, motile, non-polarized cells after EMT (23).

In the present study, we show that SW480-shE cells may be regarded as EMT-induced mesenchymal cells, and that these cells possess CSC properties. With $C D H 1$ knockdown, E-cadherin expression in SW480-shE cells decreased, accompanied by an increase in $\mathrm{N}$-cadherin and Vimentin expression. SW480-shE cells presented with disrupted intercellular adhesion and a reorganized cytoskeleton into an EMT-like formation. These cells were comparatively quiescent, grew slower and had a greater colony-formating ability, as shown by both 2D and 3D cultures, compared to the SW480-shC cells. In an in vivo experiment, SW480-shE cells also displayed stronger tumorigenic ability, which is a main characteristic of CSCs. Based on the fact that CD24 and CD44 are widely used for isolating CSCs from solid tumors, including CRC (16), we used these markers in our study $(24,25)$; our results demonstrated that the combination of these 2 markers enhanced the purity of CSCs and enriched the $\mathrm{CD} 24^{+} \mathrm{CD} 44^{+}$subpopulation significantly in the SW480-shE cells. In conclusion, SW480-shE cells possess most of the properties of CSCs.

Since CSC resistance to chemotherapy is the barrier to successful tumor treatment, a previous study used the EMT model for enriching breast CSCs, and high-throughput screening for anticancer drugs with CSC-specific toxicity (5). Generally, CSCs are obtained through FACS, spheroid culture and TGF $\beta$-induced EMT in CRC (26). However, these CSCs cannot be used for long-term culture due to differentiation. Therefore, a more effective and stable model of CSCs is required for the study of CSCs and screening for CSC-sensitive chemotherapeutic drugs.
In conclusion, SW480-shE cells possess most of the properties of EMT and CSCs, and may be a stable model for cancer study, especially for screening for CSC-sensitive drugs. With the deepening research of the molecular mechanisms involved in CRC and drug research, the prognosis of CRC may significantly improve.

\section{Acknowledgements}

The authors thank Dr Wei Yuan for providing the CDH1shRNA vector (pSilencer 4.1-CMV) and Dr Zhong Shi for language editing. This study was supported by grants from the National Natural Science Foundation of China (no. 91019005), the Zhejiang Provincial Natural Science Foundation of China (no. Y2110034), and the Zhejiang Education Department of China (no. Y200909117).

\section{References}

1. Ferlay J, Shin HR, Bray F, Forman D, Mathers C and Parkin DM: Estimates of worldwide burden of cancer in 2008: GLOBOCAN 2008. Int J Cancer 127: 2893-2917, 2010.

2. Vo DM, Julien LA and Thorson AG: Current controversies in colon and rectal cancer. Minerva Chir 65: 677-693, 2010.

3. Greenlee RT, Hill-Harmon MB, Murray T and Thun M: Cancer statistics, 2001. CA Cancer J Clin 51: 15-36, 2001.

4. Pang R, Law WL, Chu AC, et al: A subpopulation of CD26 $6^{+}$ cancer stem cells with metastatic capacity in human colorectal cancer. Cell Stem Cell 6: 603-615, 2010.

5. Gupta PB, Onder TT, Jiang G, et al: Identification of selective inhibitors of cancer stem cells by high-throughput screening. Cell 138: 645-659, 2009.

6. Visvader JE and Lindeman GJ: Cancer stem cells in solid tumours: accumulating evidence and unresolved questions. Nat Rev Cancer 8: 755-768, 2008.

7. Mani SA, Guo W, Liao MJ, et al: The epithelial-mesenchymal transition generates cells with properties of stem cells. Cell 133: 704-715, 2008.

8. Thiery JP, Acloque H, Huang RY and Nieto MA: Epithelialmesenchymal transitions in development and disease. Cell 139: 871-890, 2009.

9. Fang X, Cai Y, Liu J, et al: Twist2 contributes to breast cancer progression by promoting an epithelial-mesenchymal transition and cancer stem-like cell self-renewal. Oncogene 30: 4707-4720, 2011.

10. Prall F: Tumour budding in colorectal carcinoma. Histopathology 50: 151-162, 2007

11. Thiery JP: Epithelial-mesenchymal transitions in tumour progression. Nat Rev Cancer 2: 442-454, 2002.

12. Natalwala A, Spychal R and Tselepis C: Epithelial-mesenchymal transition mediated tumourigenesis in the gastrointestinal tract. World J Gastroenterol 14: 3792-3797, 2008.

13. Voulgari A and Pintzas A: Epithelial-mesenchymal transition in cancer metastasis: mechanisms, markers and strategies to overcome drug resistance in the clinic. Biochim Biophys Acta 1796: 75-90, 2009.

14. Du L, Wang H, He L, et al: CD44 is of functional importance for colorectal cancer stem cells. Clin Cancer Res 14: 6751-6760, 2008.

15. Hwang WL, Yang MH, Tsai ML, et al: SNAIL regulates interleukin-8 expression, stem cell-like activity, and tumorigenicity of human colorectal carcinoma cells. Gastroenterology 141: 279-291, e271-275, 2011.

16. Du L, Wang H and He L: Correction: Article on CD44 as a marker of colorectal cancer stem cells. Clin Cancer Res 14: 7964-7967, 2008.

17. Zhang $\mathrm{S}$, Balch $\mathrm{C}$, Chan $\mathrm{MW}$, et al: Identification and characterization of ovarian cancer-initiating cells from primary human tumors. Cancer Res 68: 4311-4320, 2008.

18. Marotta LL and Polyak K: Cancer stem cells: a model in the making. Curr Opin Genet Dev 19: 44-50, 2009.

19. Baumann M, Krause M and Hill R: Exploring the role of cancer stem cells in radioresistance. Nat Rev Cancer 8: 545-554, 2008. 
20. Jiang X, Gwye Y, Russell D, et al: CD133 expression in chemoresistant Ewing sarcoma cells. BMC Cancer 10: 116, 2010.

21. Ho MM, Ng AV, Lam S and Hung JY: Side population in human lung cancer cell lines and tumors is enriched with stem-like cancer cells. Cancer Res 67: 4827-4833, 2007.

22. Kong D, Li Y, Wang Z and Sarkar FH: Cancer stem cells and epithelial-to-mesenchymal transition (EMT)-phenotypic cells: are they cousins or twins? Cancers (Basel) 3: 716-729, 2011.

23. Yilmaz M and Christofori G: EMT, the cytoskeleton, and cancer cell invasion. Cancer Metastasis Rev 28: 15-33, 2009.

24. Vermeulen L, Todaro M, de Sousa Mello F, et al: Single-cell cloning of colon cancer stem cells reveals a multi-lineage differentiation capacity. Proc Natl Acad Sci USA 105: 13427-13432, 2008.
25. Choi D, Lee HW, Hur KY, et al: Cancer stem cell markers CD133 and CD24 correlate with invasiveness and differentiation in colorectal adenocarcinoma. World J Gastroenterol 15: 2258-2264, 2009.

26. Cufi S, Vazquez-Martin A, Oliveras-Ferraros C, Martin-Castillo B, Joven J and Menendez JA: Metformin against TGFbeta-induced epithelial-to-mesenchymal transition (EMT): from cancer stem cells to aging-associated fibrosis. Cell Cycle 9: 4461-4468, 2010. 